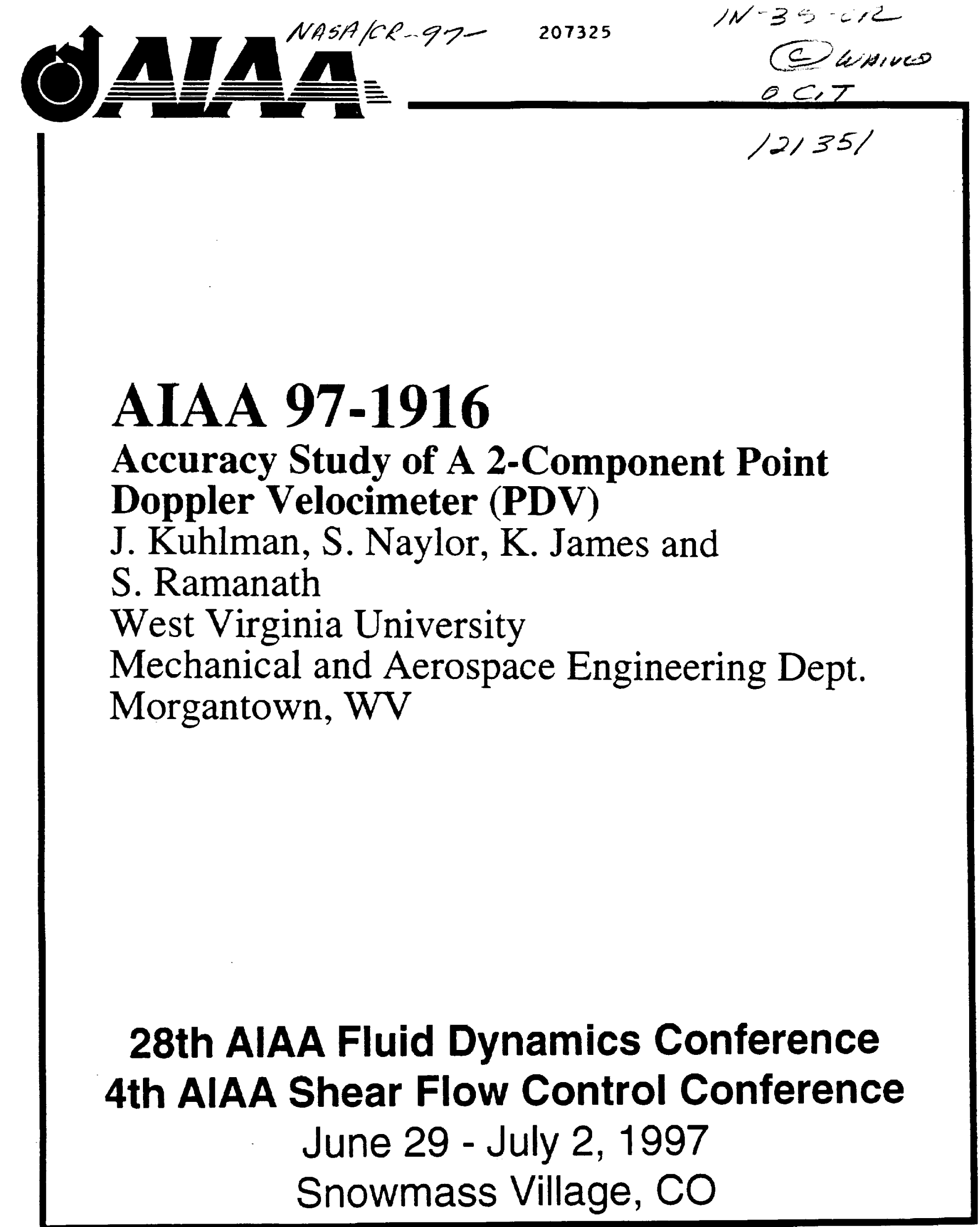

For permission to copy or republish, contact the American Institute of Aeronautics and Astronautics 1801 Alexander Bell Drive, Suite 500, Reston, VA 20191- 4344 


\title{
ACCURACY STUDY OF A 2-COMPONENT POINT DOPPLER VELOCIMETER (PDV)
}

\author{
John Kuhlman ${ }^{1}$, Steve Naylor ${ }^{2}$, Kelly James ${ }^{2}$, and Senthil Ramanath ${ }^{2}$ \\ West Virginia University \\ Mechanical and Aerospace Engineering Department \\ Morgantown, WV 26506-6106
}

(304) 293-3111

\begin{abstract}
A two-component Point Doppler Velocimeter (PDV) which has recently been developed is described, and a series of velocity measurements which have been obtained to quantify the accuracy of the PDV system are summarized. This PDV system uses molecular iodine vapor cells as frequency discriminating filters to determine the Doppler shift of laser light which is scattered off of seed particles in a flow. The majority of results which have been obtained to date are for the mean velocity of a rotating wheel, although preliminary. data are described for fully-developed turbulent pipe flow.

Accuracy of the present wheel velocity data is approximately $\pm 1 \%$ of full scale, while linearity of a single channel is on the order of $\pm 0.5 \%$ (ie, \pm 0.6 $\mathrm{m} / \mathrm{sec}$ and $\pm 0.3 \mathrm{~m} / \mathrm{sec}$, out of $57 \mathrm{~m} / \mathrm{sec}$, respectively). The observed linearity of these results is on the order of the accuracy to which the speed of the rotating wheel has been set for individual data readings. The absolute accuracy of the rotating wheel data is shown to be consistent with the level of repeatability of the cell calibrations.
\end{abstract}

The preliminary turbulent pipe flow data show consistent turbulence intensity values, and mean axial velocity profiles generally agree with pitot probe data. However, there is at present an offset error in the radial velocity which is on the order of $5-10 \%$ of the mean axial velocity.

\section{INTRODUCTION}

This research project is exploring the accuracy of Doppler Global Velocimetry (DGV), a non-

Copyright (01997 by the American Institute of Aeronautics and Astronautics, Inc. All rights reserved.

'Professor, Associate Fellow AIAA

${ }^{2}$ Graduate Research Assistant intrusive, planar imaging, Doppler-based velocimetry technique, as well as the accuracy of related Point Doppler Velocimetry (PDV). Both of these techniques use an iodine vapor cell absorption line filter (ALF) to determine the Doppler shift, and hence the velocity, of small seed particles in a flow field, as these particles pass through a twodimensional sheet of laser light. The same portion of the light sheet is viewed through a beam splitter, either by a pair of video cameras (for DGV), or a pair of photodetectors (for PDV), with the iodine cell ALF placed in the optical path of one of the cameras or photodetectors. Laser wavelength and ALF absorption band are matched such that the range of flow velocities of interest yields Doppler shifted frequencies which lie in the linear portion of the absorption band of the AIF. As a result, the ratio of the light intensities seen by the two detectors at a point in the flow yields a signal which is proportional to the particle velocity.

For a non-scanned "point" PDV system, very high data rates are possible, limited primarily by seeding/signal strength and AV conversion speed. Accuracy of such a point system may be comparable to that of corventional laser velocimetry (LV). Use of conventional CCD cameras to view a region of the light sheet yields velocity data at a typical resolution of 640 pixels by 512 lines, at framing rates of up to standard video rates of 30 frames per second, but at a reduced accuracy (typically on the order of about \pm 5 to $8 \%$ ). This reduced accuracy is primarily due to camera noise; cooled cameras can reduce this error, but at a significant increase in cost. Also, framing rates are typically reduced to on the order of $1 \mathrm{~Hz}$ for these more accurate, cooled cameras.

A two-component non-scanned point PDV system has been developed to date in the current project A two-component scanned DGV system using $C C D$ cameras is currently also under development. The accuracy limits of both systems are being systematically explored, through a series of 
measurements in relatively simple, unheated flows such as fully-developed turbulent pipe flow, a turbulent circular jet, and grid turbulence. A rotating wheel is also being used as a velocity standard. The present paper describes the twocomponent PDV system, and presents typical velocity measurements which have been obtained for a rotating wheel, to assess the accuracy of the PDV system for mean velocity measurements. Also, preliminary PDV measurements in the fullydeveloped pipe flow are presented.

\section{LITERATURE SUMMARY}

Several different non-intrusive whole field velocimetry techniques are currently under development which provide velocity data in a plane, which can thus greatly recuce the time required to map out a complex flow field. It is expected that this can lead to enhanced insight into flow physics. Of these techniques, particle image velocimetry (PIV) has perhaps been the most fully developed (Adrian and Yao, 1983). Scalar imaging velocimetry (SIV) shows promise for determination of three dimensional velocity data in large Schmidt number liquid flows (Dahm, 1992). Another nonintrusive technique under development by Miles (1992) is the RELIEF technique, which also appears to be limited to two velocity components in a plane, similar to PIV.

A fourth concept for acquiring non-intrusive real-time velocity measurements in a planar region called Doppler global velocimetry (DGV) has been patented by Komine (1990). This technique uses a pair of video cameras and an iodine vapor cell absorption line filter (ALF) for each velocity component, to measure the average Doppler frequency shift, averaged over each pixel, of the light scattered off minute seed particles in a flow as they pass through a planar sheet of laser light. This new DGV velocity measurement technique is currently being developed into an accurate instrument by a group at the NASA Langley Research Center (Meyers et al.,1991). This same group has also funded work at Northrop (Meyers and Komine, 1991).

Others are also developing related DGV concepts; for example, Miles et al (1991) have developed a filtered Rayleigh scattering (FRS) technique which shows potential for providing nonintrusive velocity measurements without requiring any seeding. An optically thick ALF is used to filter out all signal but the Doppler shifted frequencies due to molecular Rayleigh scattering.

A preliminary study has been conducted by Hoffenberg and Sullivan (1993) to measure the Doppler frequency, and hence velocity, at a point using a non-scanned filtered particle scattering (FPS) technique. Measurement accuracy of both mean and turbulence quantities was comparable to corventional LV data at the exit of an axisymmetric jet at about $100 \mathrm{ft} / \mathrm{sec}$. However, large errors in mean and RMS velocities were found near the edges of the jet, possibly due to uneven seeding and low signal-to-noise ratio. Similar point DGV studies have been conducted by Morrison et al (1994), and by Roehle and Schodl (1994). Roehle and Schodl have improved the accuracy of their measurements through active stabilization of the frequency of their $\mathrm{CW}$ Argon ion laser.

Recently, others at NASA Ames (McKenzie, 1995), NASA Langley (Smith and Northam, 1995), and Ohio State University (Ellioth, et al, 1994, and Clancy and Samimy, 1997) have also developed scanned DGV systems. Both Smith and McKenzie have used a single video camera to record both the reference and signal images for each velocity component; this split-image technique reduces resolution by a factor of two, but also reduces system cost and complexity. The data by McKenzie for single channel point measurements on a rotating wheel (1995) display the best absolute accuracy (on the order of $\pm 1.2 \mathrm{~m} / \mathrm{sec}$ ) of any measurements to date. McKenzie's more recent (1997) planar imaging results of the velocity of the same rotating wheel displayed a lower level of accuracy $( \pm 2-5$ $\mathrm{m} / \mathrm{sec}$ ).

Thus, it is clear that in a very short time (approximately six years), DGV has developed to a point where capability has been demonstrated for making non-intrusive mean flow velocity vector measurements in a plane, in a variety of complex single phase flow fields of practical significance. While current scanned DGV systems lack the accuracy or resolution of corventional LV systems or PIV (to date, documented as on the order of from 5 to $8 \%$, versus $1 \%$ for $L V$, at about $100 \mathrm{ft} / \mathrm{sec}$.), DGV has proven in a very short time to be an extremely flexible whole-field velocimetry technique.

Following the nomenclature of McKenzie (1995), the basic equation relating the Doppler shif 
frequency to the resolved velocity component is given by $\delta v=(\underline{\mathbf{0}}-\underline{\mathbf{i}}) \bullet \underline{V} / \lambda$, where $\delta v$ is the Doppler frequency, $\underline{V}$ is the velocity vector, $\lambda$ is the incident laser frequency, and $\underline{0}$ and $\mathbf{i}$ are the observer and laser propagation directions, respectively (see Fig. 1). Thus, the resolved velocity component is in the direction of the sum of $\underline{q}$ and $(-\mathbf{i})$.

\section{APPARATUS AND PROCEDURE}

The present point PDV system closely follows the basic DGV configuration using two inch diameter iodine cells which was originally developed by Meyers et al. (1991), except that photodiodes are currently being used, along with front lenses and pinholes, to collect scattered light from a single point in a seeded flow which is illuminated by a CW Argon ion laser. Laser frequency has not been actively controlled, but instead a reference iodine cell has been used to compensate for any changes (due to laser frequency drift) in the voltage ratios for the iodine cells which view the flow and receive the Doppler shifted scattered light. A laser spectrum analyzer has been used to monitor laser mode shape and detect the occurrence of mode hops. After a suitable warm-up period for the laser and spectrum analyzer (typically on the order of one hour, to achieve optimum frequency stability), frequency drift on the order of $50 \mathrm{MHz}$ has been observed over a time period on the order of 30 minutes; this is close to the resolution of the spectrum analyzer and the claimed frequency stability for the laser.

This reference iodine cell system and the Argon ion laser are shown in Fig. 2, along with the laser spectrum analyzer and Argon ion laser. Neutral density filters and a beam expander are used to ensure that the iodine cell is not saturated by the reference beam. A quarter wave plate has been used to circularly polarize the laser light which is used to illuminate the flow. The layout of one of the two PDV channels is shown in Fig. 3, while a schematic of how the entire system has been configured for the wheel velocity measurements may be found in Fig. 4. The two PDV channels include pinholes behind each front lens, as implemented by Roehie and Schodl (1994); these pinholes act as spatial filters to set the size of the sensing region in the light sheet, as well as to reduce the effects of secondary scattering by limiting the depth of field. Planocorvex lenses have been installed in front of the photodiodes (see Fig. 3), to ensure that all scattered light from the sensing region is imaged onto the photodetectors. Also, improvements in accuracy have been obtained by carefully optimizing and matching amplifier gains for each pair of photodiodes, as well as by enclosing each PDV channel to reduce background scattered light intensities.

Achieving adequate temperature stability of the side arms of the iodine cells has been found by researchers at NASA Langley to be an essential requirement for accurate operation of a DGV system. A temperature control system which is similar to those used by NASA Langley has been implemented in the present system, which is comprised of a pair of electrical band heaters which heat a hollow bushing made from oxygen-free copper, which surrounds the iodine cell except for the two optical windows and the side arm. The side arm has been thermally "grounded" by a copper wire which is bonded to the tip of the side arm, and then bolted to the optical breadboard on which the DGV system is mounted. The entire system has been enclosed in an insulated box (as shown in exploded view in Fig. 3) to shield the cell from air currents or room temperature variations, and the optical windows of the cell have been insulated from the room air by phenolic tubes fitted with additional AR-coated crown glass windows, which protrude through the sides of the aluminum box. This creates a heated dead air space next to the outside of the cell by the optical windows and prevents the formation of solid iodine crystals on the optical windows. The Omega PD temperature controller has been adjusted to achieve very stable operation, where the copper sheath surrounding the iodine cell typically operates at a temperature which is nominally $10^{\circ} \mathrm{C}$ above the side arm temperature. This ensures that all solid phase iodine collects in the side arm of the cell. Cells have been operated at stem temperatures of $45^{\circ} \mathrm{C}$, since absorption well slope is a maximum there (McKenzie, 1995).

Data acquisition software has been developed in Visual Basic 4.0 to allow continuous monitoring and data acquisition of the cell temperatures, for the reference cell and each of the cells used in the two PDV channels. Long term drift in iodine cell temperature has been measured to be on the order of $\pm 0.1^{\circ} \mathrm{C}$ (the specified set point accuracy of the temperature controller), once the cell has warmed up to its steady operating temperature. Short term fluctuations have been measured which are on the order of $0.03-0.04{ }^{\circ} \mathrm{C}$; this approaches the resolution of our 16 bit thermocouple $A D$ board. 
To determine the accuracy of the twocomponent PDV system, a rotating wheel apparatus has been developed, consisting of a 12 inch diameter, anodized circular aluminum disk which has been painted white, and mounted on a variable speed DC motor. This wheel can achieve tip velocities of approximately $\pm 28 \mathrm{~m} / \mathrm{sec}$. A second wheel, consisting of a 6 inch diameter lexan disk mounted to a Dremel tool can achieve tip speeds of about $100 \mathrm{~m} / \mathrm{sec}$, but this apparatus has not been used in the present study due to excessive wobble. In addition, a calibration procedure similar to that which has been used by NASA Langley personnel, where the laser is mechanically mode hopped by tilting the etalon has been utilized to calibrate the iodine cells.

A 1.5 inch diameter, fully-developed turbulent pipe flow apparatus has been developed, as has a small grid turbulence flow facility. Also, a jet facility is available which has interchangeable convergent nozzles with exit diameters of $0.375,0.5$, and 1.0 inches, and which can attain exit velocities up to $120 \mathrm{~m} / \mathrm{sec}$, with very low exit turbulence levels. A conventional LV data set for this jet, as well as for a companion annular jet, has been given by Kuhlman (1994). Flow seeding has been achieved using a commercial fog machine in the present work. A second technique using a soldering iron to vaporize the fog fluid is also available (McKenzie, 1995).

A computer-controlled, three-axis traversing system has been developed (Fig. 5), as described in the thesis by Ramanath (1997), for use in positioning the flow facilities with respect to the fixed, two channel PDV system, so that velocity contours may be mapped out in a plane or volume. This traverse allows movement in a volume which is two feet by a foot and a half in a horizontal plane, by one foot in the vertical direction. Accuracy of a single traverse move has been found to be better than $0.001^{\prime \prime}$ for typical moves on the order of a few inches (Ramanath, 1997).

An 8 channel, 16 bit, simultaneous-sampleand-hold IOTech $A / D$ board is used for digital data acquisition of the photodetector output voltages for the reference iodine cell, and for the two PDV channels. The RMS noise level for this board is \pm $0.3 \mathrm{mV}$ on a 10 volt scale. Windows-based data acquisition software has been developed (again, using Visual Basic) for this board. In addition, companion VB data reduction programs have been developed, to automate the data reduction process.

Calibration of the iodine cells has been accomplished using a continuous scan of the mode structure of the Argon ion laser, by mechanically altering the tilt of the etalon through about 10-20 mode hops, over a 20-30 second period. A typical time history of the voltage ratios for the reference and the two signal channels for this process is shown in Fig. 6. It is generally noted that the signal-toreference voltage ratio for each iodine cell varies continuously between mode hops. Occurrence of mode hops has been detected by a sudden jump in reference photodiode voltage. The ratio value for any one mode hop may be computed as an average value, the value at the left end of that mode, or the value at the right end of the mode; the best results have been obtained using the average value (James, 1997). It has been found that this continuous scan mode hop calibration technique offers better accuracy than an earlier technique, where individual ratio values were measured after each mode hop of the laser. This is because the cell temperatures cannot change significantly over the 20-30 second time period required to perform a scan. Also, the effects due to variability of where one stops the mechanical screw adjust on the etalon tilt screw are minimized by this technique. Significant further improvement in calibration accuracy has also been obtained by averaging several of these individual continuous scan mode hop calibrations together. This improved calibration consists of several (from 3 to 6) continuous mode hop calibration data sets for each cell. A singie cell calibration data file is formed by "sliding" all mode hop calibrations for any one cell, to overlay them on one arbitrarily-selected calibration of the set. This procedure is accomplished by linear interpolation, and is necessary because of laser drift between mode hop calibrations, where the ratio value for the $n^{\text {th }}$ mode hop for any one cell will change, especially when the room temperature varies significantly.

Eight bit Hitachi CCD cameras and a Matrox Genesis frame grabber are being used for the two component DGV system, which is currently under development. Since both a 2-component point PDV system as well as a 2-component imaging DGV system will eventually be available, it will be possible to make direct comparisons between the PDV measurements and the scanned DGV measurements in the same flow fields, measured from the same viewing directions, with comparable smoke particle size and laser illumination levels. 
This will provide direct experimental documentation of the amount of additional error introduced by the $C C D$ video cameras.

\section{RESULTS}

Early data repeatability, as documented in the thesis by Ramanath (1997), was poor. The standard deviation of the slopes of plots of the measured PDV velocity versus the known velocity of a rotating wheel was on the order of 8-15\%, even though the linearity of each individual data set was quite good (on the order of $\pm 1-2 \mathrm{~m} / \mathrm{sec}$, out of $58 \mathrm{~m} / \mathrm{sec}$ ). Similar results were initially obtained by James (1997). However, the improved cell calibration procechures described above have significantly increased the accuracy of the present PDV system.

Typical examples of the present results for the rotating wheel will now be presented; these data are presented in much more detail in the thesis by James (1997). Data has been acquired in two different fashions: first, the 2-component PDV system was configured so that we could acquire two simultaneous, but independent, measurements of the wheel velocity magnitude, from two slightly different viewing directions. To do this, the system was set up so that both PDV channels had relatively good sensitivities in the wheel velocity direction. One channel (channel 2) was set up with a viewing direction which was at an angle of approximately 42 degrees from the laser propagation direction. The other channel (channel 1) viewred the wheel at an angle of approximately 75 degrees, by imaging the wheel off of a mirror which was mounted on the breadboard which held the channel 2 optical components (see Fig. 4). The wheel was inclined slightly (about 5 degrees) to the laser propagation direction. In this configuration, the resulting velocity data have been converted to wheel velocities by assuming that the direction of the wheel velocity was known. In the second configuration, the two PDV channels were set up with widely differing viewing angles (approximately 126 degrees from the laser propagation direction for channel 1 , and approximately 42 degrees for channel 2), and the two PDV velocity measurements were used to resolve orthogonal $x$ - and $y$-velocity components, from which the wheel velocity magnitude was computed as the square root of the sum of the squares of the components.

With the 2-component PDV system set up in the first configuration, a series of seven wheel velocity data sets were acquired, all using a single cell calibration data ru (James, 1997). A typical example of the resulting data is shown in Fig. 7, where the measured PDV velocity magnitudes for channels 1 and 2 are shown plotted against the known wheel omega-r. Observed linearity is quite good. The standard deviations of the slopes of the linear curve fit equations from the correct slope of exactly 1.0 were calculated for several different curve fit options for each of the seven data sets. These errors ranged from a maximum of about \pm 15 $\%$ for a linear curve fit of the cell calibrations (on the same order of error as for the earlier data), to a low of $\neq 1-2 \%$ for a fourth-order curve fit. Specifically, from the individual slope results listed in Table 1 , the deviations of the measured sensitivities were 1.4 $\%$ for channel 2 and $2.3 \%$ for channel 1 using fourth- order curve fits to the calibration data. The accuracy to which the viewing angle could be measured was less for the channel which viewed the wheel off of the mirror (channel 1); this is believed to be the explanation for the larger error in sensitivity or slope for this channel. The actual standard deviations of the slope values are somewhat smaller, indicating that there is some bias error in these results; channel 2 slopes have a standard deviation of $1.1 \%$, while channel 1 slopes have a standard deviation of $1.5 \%$. Since the total range of wheel velocity for these measurements is about 57 $\mathrm{m} / \mathrm{sec}$, these observed $1-2 \%$ errors correspond to velocity error magnitudes of approximately $\pm 0.6-1.2$ $\mathrm{m} / \mathrm{sec}$, which is quite good. Also, the standard deviations of the actual PDV velocity data points from the least squares linear curve fits have been computed, as listed in Table 1, and these errors are even smaller than the slope errors. For channel 2, the data for all seven runs display a standard deviation from a linear fit of $0.7 \%( \pm 0.4 \mathrm{~m} / \mathrm{sec})$, while channel 1 displays a standard deviation from a linear fit of $0.5 \%( \pm 0.3 \mathrm{~m} / \mathrm{sec})$. Again, this level of linearity is quite good. Since the errors in the slopes of the measured velocity versus omega-r were smallest using the fourth-order polynomial curve fits to the average ratio data, all subsequent data has been reduced using this method. It has been observed that the reference cell is not able to consistently determine the zero velocity; thus, for the present results zero velocity has been fixed by a measurement of all voltage ratio values just prior to and after the actual data acquisition. Other researchers using scanned systems have had similar problems, which they have addressed by imaging a zero velocity region somewhere in each camera image (McKenzie, 1996, and Reinath, 1996; 
personal communication).

With the 2-component PDV system set up in the second configuration, a series of twelve wheel velocity data runs have been acquired, using five different cell calibrations (James, 1997). An example of this data is shown in Fig. 8, while the individual orthogonal velocity measurements are shown for this run in Fig. 9. The individual slopes and standard deviations of the data from a linear fit for each nun have been given in Table 2. Here the standard deviation of the slopes of the linear curve fits to the data is $1 \%$, and the data points exhibit deviations from the linear curve fits with a standard deviation of $1.1 \%( \pm 0.65 \mathrm{~m} / \mathrm{sec})$. Again, this is felt to be quite good accuracy. The accuracy of the channel 1 data, which is less sensitive to the wheel velocity, is not as good as that of channel 2. As a result, the accuracy of the computed $x$-velocity component, normal to the laser propagation direction, is not as good as the accuracy of the computed y-velocity component (Fig. 9). The correct sensitivities to the $x$ - and $y$-velocity components, as shown in Fig. 9, were $\cos \left(5^{\circ}\right)$ and $-\sin \left(5^{\circ}\right)$.

During efforts to improve the accuracy of the point DGV system, typical RMS fluctuation levels of the voltage signals from the photodiodes have been monitored, along with the RMS fluctuation levels of the computed ratio of signal-to-reference voltages.

For the reference system, RMS voltage fluctuations typically are on the order of $0.5 \%$ of the mean voltage for each photodiode, but the RMS fluctuation in the ratio is approximately $0.2 \%$ of the mean ratio value. Similar percentage fluctuations in the ratio value have been observed during experiments using the rotating wheel. Recently, a simple math model of this phenomenon has been proposed by Ramanath, where the individual signals from the photodiodes are modeled as sine functions, each of which can have offset and/or phase errors. The ratio of these two model signals can show increased, "spiky" fluctuation levels, as is sometimes observed in the data when the raw voltage levels are small. Offset errors may occur due to inaccuracies or changes in the detector dark or background voltages; such errors become more significant as the signal level decreases.

An analysis of the major error sources for the present results has been performed, as very briefly summarized below.

1. The accuracy to which the rotating wheel speed has been set has been checked by using a strobe and measuring the time between voltage spikes in the output of one of our PDV signal photodiodes, using an oscilloscope. The reading error for these time measurements was estimated to be no greater than $\pm 0.5 \%$, which is about the same as the observed accuracy to which the individual PDV data points fit to a least-squares straight line. To do any better than this at checking the linearity of response of our instrument, the individual wheel speed settings would need to be measured with greater accuracy, for each data point.

2. The level of zero velocity drift of our 2component PDV system has been measured for a period of 30 minutes, as shown in the Fig. 10. The observed drift in zero velocity is on the order of \pm 1.5 $\mathrm{m} / \mathrm{sec}$; this is thought to be primarily due to drift in cell stem temperatures, as shown in Fig. 11, where the difference between the stem temperature for the PDV signal channel, and the reference cell stem temperature, is shown for each channel, for the same time period as the zero velocity data run (Fig. 10) The similarity of the shapes of these two graphs indicates that this error is due primarily to the combination of the accuracy of the cell stem temperature controllers and the accuracy of the calibration curves. It is also felt that this drift in cell stem temperature difference is a significant contributor to the observed error in sensitivity of the two PDV channels. However, no correlation has been found between the slope data given in Tables 1 and 2, and the stem temperature difference values for each data run. It is noted that the present zero velocity drift $( \pm 1.5 \mathrm{~m} / \mathrm{sec})$ is reduced relative to the earlier results presented by Ramanath (1997), of \pm 4 $\mathrm{m} / \mathrm{sec}$; this is due to the improved accuracy of the present cell calibrations.

3. Using four of the five calibration curves which have been used to rectuce the second series of wheel velocity data, the repeatability of the calibration curves from day-to-day has been investigated (James, 1997), by forcing pairs of corresponding calibration curves to overlay exactly at the middle of the ratio range which was actually used to reduce the wheel velocity data, and computing the difference in computed velocities which would result at the top and bottom of these ratio ranges if the two different cell calibrations were used to rednce the same wheel velocity data set. (See Fig. 12 for an example.) The resulting errors were found to be between 2.3 and $0.3 \mathrm{~m} / \mathrm{sec}$, with and average error of $0.7 \mathrm{~m} / \mathrm{sec}$. ( $\pm 1.2 \%$ ). These errors 
due to using different cell calibrations are on the same order as the observed error in slopes of the wheel velocity data results. As a result, it is believed that while the current cell calibration procedure has led to a significant improvement in our resuits, the primary error source in the current PDV system is still the accuracy of the cell calibrations. To improve this accuracy further, work is underway to implement the curve-fitting procedure described by McKenzie (1995), which uses the theoretical iodine cell absorption curve calculated by the Forkey theoretical cell absorption model to curve fit the actual cell calibration data. It is felt that this procedure will remove the judgment which is required in the current polynomial curve fit technique, where the user must decide which portion of the cell calibration data to include in the curve fit process. Also, from Fig. 12 it is noted that the useful range of the developed PDV system is on the order of $100 \mathrm{~m} / \mathrm{sec}$; this range could be increased by the addition of a neutral buffer gas to the iodine vapor (Elliott, et al., 1994). A recuction in this useful range, in hopes of increasing the resolution of the Doppler velocimeter, may be possible through use of a Cesium Faraday filter (Bloom, et al., 1993).

An example of preliminary two-component PDV data obtained from a traverse across the exit of the fully-developed pipe flow apparatus, at a nominal Reynolds number of 76,000 , is shown in Figs. 13 and 14. These data have been obtained at a sampling rate of $5 \mathrm{kHz}$, with a data record length of $10^{4}$ points ( 2 seconds) at each measurement location. The axial mean velocities agree reasonably well with results from a pitot tube survey, but radial mean velocities currently display an offset error on the order of $2-4 \mathrm{~m} / \mathrm{sec}$ (Fig. 13). Tubulent velocities (Fig. 14) agree well with the hot wire data of Laufer (1954). There are significant difficulties near the pipe walls, both due to reduced signal-to-noise levels due to less smoke, as well as to reflections of the scattered light off of the pipe walls. However, at present the greatest difficulty appears to be obtaining accurate, reliable zero velocity ratio values.

\section{CONCLUSIONS}

A two-component Point Doppler Velocimeter (PDV) system has been developed, and its accuracy has been investigated by measuring the velocity of a rotating wheel. Also, preliminary two-component PDV velocity data have been presented for a fullydeveloped turbulent pipe flow.
Accuracy of the present PDV system, based on the rotating wheel velocity results, has been documented to be on the order of $\pm 0.6 \mathrm{~m} / \mathrm{sec}$ over a velocity range of $57 \mathrm{~m} / \mathrm{sec}$ (ie, approximately $\pm 1 \%$ of full scale). Linearity of the present PDV system, again based on the wheel data, has been documented to be on the order of $\pm 0.3 \mathrm{~m} / \mathrm{sec}$, which is on the order of the accuracy of the individual wheel velocity settings; this linearity is on the order of $0.5 \%$ of the measured velocity range. Both of these observed accuracy measurements are considerably better than those documented to date by other researchers.

Preliminary two-component PDV velocity data have been presented for a fully-developed turbulent pipe flow, at a Reynolds number of approximately 76,000 . Turbulence intensity values agree well with earlier hot wire data, and mean axial velocity data agree reasonably well with pitot tube results. However, radial velocity results show a consistent offset error which is on the order of ten percent of the mean axial velocity; the reasons for this error are at present uncertain, but are believed to be due to inaccuracy in the determination of zero velocity.

It is planned that the developed two-component PDV instrumentation will be utilized to obtain further velocity data in the fully-developed turbulent pipe flow and axisymmetric jet flow facilities. These data will be compared with conventional LV data. Similar studies are also planned using the twocomponent scanned DGV system which is currently under development. The ultimate goal of the present research is to optimize the system accuracies for both the point PDV and scanned DGV systems, and then to carefully document the accuracies of both optimized systems, and quantify the dominant error sources for each.

\section{ACKNOWLEDGMENTS}

The present work is being supported under AFOSR/DEPSCOR Grant F49620-94-1-0434, Dr. James M. McMichael, technical monitor. Senthil Ramanath received partial support under NASA grant NAGW-4464, The authors are grateful for the technical assistance of Jim Meyers, Joe Lee, Rich Schwart, Angelo Cavone, and Gary Fleming at NASA Langley Research Center, and Bob McKenzie and Mike Reinath at NASA Ames Research Center.

\section{REFERENCES}

Adrian, RJ. and Yao, C. S., "Development of 
Pulsed Laser Velocimetry for Measurement of Turbulent Flow," Proc. of the Eighth Biennial Symposium on Turbulence, Univ. of Missouri, Rolla, 1983, pp. 170-186.

Bloom, S. H., Searcy, P. A., Choi, K., Kremer, R, and Korevaar, E., "Helicopter Plume Detection by Using an Ultranarrow-Band Noncoherent Laser Doppler Velocimeter," Optics Letters, Vol. 18, No. 3, Feb. 1993, pp. 244-46.

Clancy, P. S. and Samimy, M., "MultipleComponent Velocimetry in High Speed Flows Using Planar Doppler Velocimetry," paper AIAA-97-0497, AIAA $35^{\text {th }}$ Aerospace Sciences Meeting, Jan. 6-10, 1997, Reno, NV.

Dahm, W.J.A., "Scalar Imaging Velocimetry Studies of Turbulent Flow Structure and Dynamics," AFOSR/ONR Grantee and Contractors Meeting on Turbulence Research, Chicago, II, June 10-12, 1992; pp. 73-76 of Workshop Proceedings.

Elliot, G.S., Samimy, M, and Arnette, S.A., "Details of a Molecular Filter-Based Velocimetry Technique," paper AIAA - 94-0490, AIAA 32nd Aerospace Sciences Meeting, Jan. 10-13, 1994, Reno, NV.

Forkey, J.N., Lemper, W.R., Bogdonoff, S.M, Miles, RB., and Russell, G., "Volumetric Imaging of Supersonic Boundary Layers Using Filtered Rayleigh Scattering Background Suppression," paper AIAA-94-0491,1994, Reno, NV.

Hoffenberg, R and Sullivan, J. P., "Filtered Particle Scattering: Laser Velocimetry Using an Iodine Filter," ASME Fluids Engineering Division Summer Meeting, Washington, DC, June 20-24, 1993.

James, K., "Determination of the Accuracy of a Two-Component Point Doppler Velocimetry System," MS Thesis, West Virginia University, MAE Department, 1997.

Komine, H., U.S. Patent No. 4, 919, 536 Apr. 24, 1990.

Kuhlman, J.M., "Turbulence Measurements in Annular Jets Using Laser Velocimetry", ASME Symp. Laser Anemometry: Advances and Applications, Lake Tahoe, NM, June 19-23, 1994.

Laufer, J., "The Structure of Turbulence in Fully Developed Pipe Flow," NACA TR 1174, 1954.

McKenzie, RL., "Measurement Capabilities of Planar Doppler Velocimetry Using Pulsed Lasers," paper AIAA-95-0297, AlAA 33rd Aerospace Sciences Meeting, Jan. 9-12, 1995, Reno, NV.

McKenzie, R. L., "Planar Doppler Velocimetry Performance in Low-Speed Flows," paper AIAA-970498, AIAA $35^{\text {th }}$ Aerospace Sciences Meeting, Jan. 6-10, 1997, Reno, NV.
Meyers, J.F. and Komine, H., "Doppler Global Velocimetry: A New Way to Look at Velocity," $4^{\text {th }}$ International Conference on Laser Anemometry, Advances and Applications, Aug. 5-9, 1991, Cleveland, OH, pp. 289-296 of Conference Proceedings.

Meyers, J.F., Lee, J.W., and Cavone, A. A., "Signal Processing Schemes for Doppler Global Velocimetry," $14^{\mathrm{th}}$ International Congress on Instrumentation in Aerospace Simulation Facilities, Rockville, MD, Oct. 27-31, 1991.

Miles, RB., "RELIEF Measurements of Turbulence," AFOSR/ONR Grantee and Contractors Meeting on Turbulence Research, Chicago, IL, June 10-12, 1992; pp. 62-64 of Workshop Proceedings.

Miles, R.B., Lempert, W.R, and Forkey, J., "Instantaneous Velocity Fields and Background Suppression by Filtered Rayleigh Scattering," paper AIAA-91-0357, AIAA 29th Aerospace Sciences Meeting, Jan. 7-10, 1991, Reno, NV.

Morrison, G. L., Gaharan, C.A., and DeOtte, R E., Jr., "Doppler Global Velocimetry: Problems and Pitfalls," Symposium on Laser Anemometry: Advances and Applications, June 19-23, 1994, Lake Tahoe, NV, and published in ASME FED Vol.191, pp. 1-8.

Ramanath, S., "Development of a Point Doppler Global Velocimeter (DGV)," MS Thesis, West Virginia University, MAE Department, 1997.

Roehle, I. and Schodl, R, "Evaluation of the Accuracy of the Doppler Global Technique," paper C485/046, Seminar on Optical Methods and Data Processing in Heat and Fluid Flow, Apr. 14-15, 1994, City Univ., London, UK.

Smith, M.W. and Northam, G. B., "Application of Absorption Filter-Planar Doppler Velocimetry to Sonic and Supersonic Jets," paper AIAA-95-0299, AIAA 33rd Aerospace Sciences Meeting, Jan. 9-12, 1995, Reno, NV.

Table 1 Slope data for rotating wheel, set up with mirror and using known velocity direction

$\begin{array}{ccc}\text { Run } & \begin{array}{c}\text { Slope } \\ \text { (Ch 1, Ch 2) }\end{array} & \begin{array}{c}\text { Deviation from linear fit } \\ \text { (Ch 1, Ch 2, in m/sec) }\end{array} \\ & & \\ 1 & 1.0073,1.0270 & 0.19,0.26 \\ 2 & 0.9775,0.9916 & 0.22,0.35 \\ 3 & 0.9876,1.0090 & 0.29,0.40 \\ 4 & 0.9861,0.9988 & 0.33,0.50 \\ 5 & 0.9603,1.0046 & 0.37,0.45 \\ 6 & 0.9747,1.0019 & 0.24,0.46 \\ 7 & 0.9911,1.0142 & 0.35,0.26\end{array}$


Table 2 Slope data for rotating wheel; two-channel PDV set up

$\begin{array}{ccr}\text { Run } & \text { Slope } & \begin{array}{c}\text { Deviation } \\ \text { linear fit (m }\end{array} \\ 1 & 0.9808 & 0.67 \\ 2 & 0.9981 & 0.50 \\ 3 & 0.9941 & 0.63 \\ 4 & 1.0073 & 1.34 \\ 5 & 1.0013 & 0.45 \\ 6 & 1.0080 & 0.99 \\ 7 & 1.0214 & 0.24 \\ 8 & 0.9996 & 0.35 \\ 9 & 0.9961 & 1.03 \\ 10 & 0.9928 & 0.42 \\ 11 & 0.9904 & 0.50 \\ 12 & 0.9989 & 0.69\end{array}$

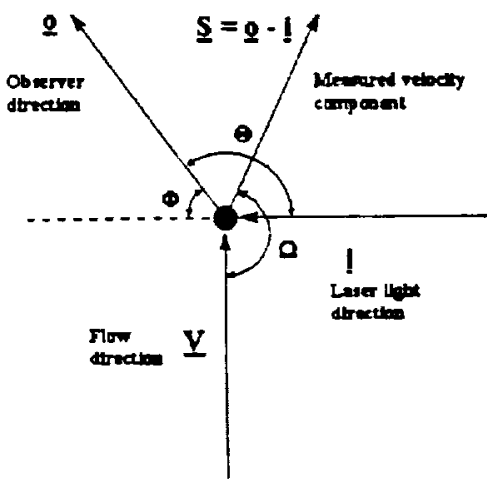

Fig. 1 Determination of Point Doppler Velocimetry (PDV) Doppler shift frequency

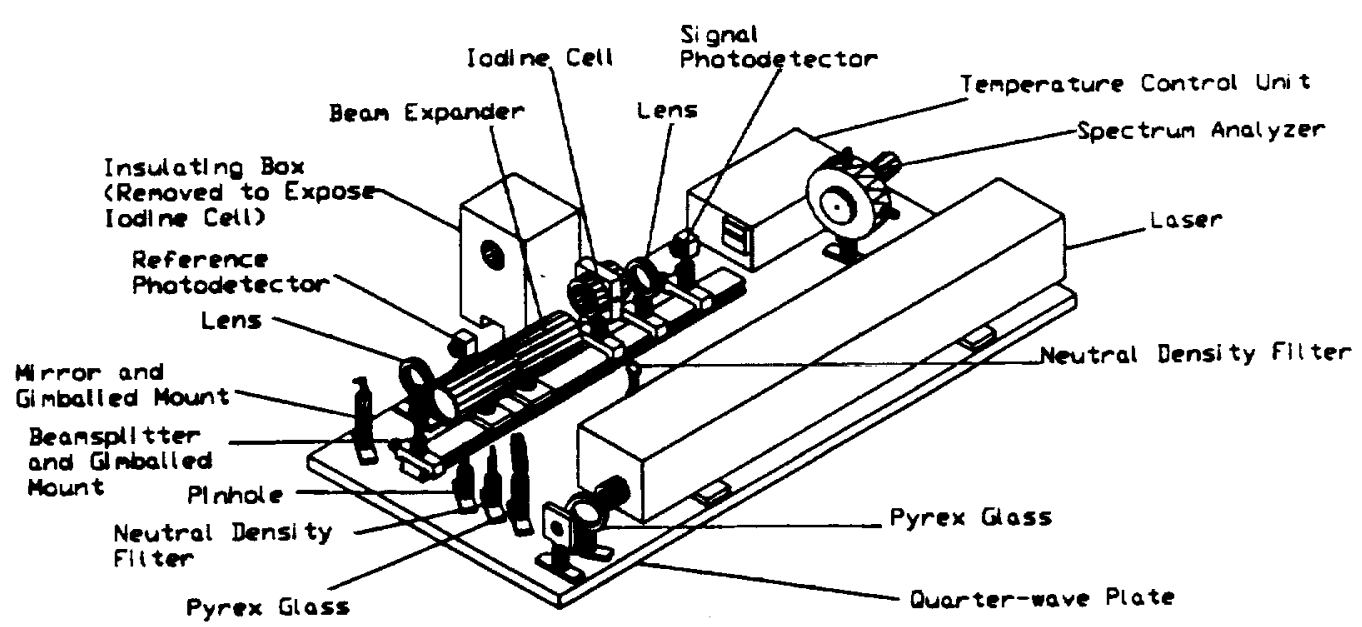

Fig. 2 Apparatus for reference iodine cell system, laser, and spectrum analyzer

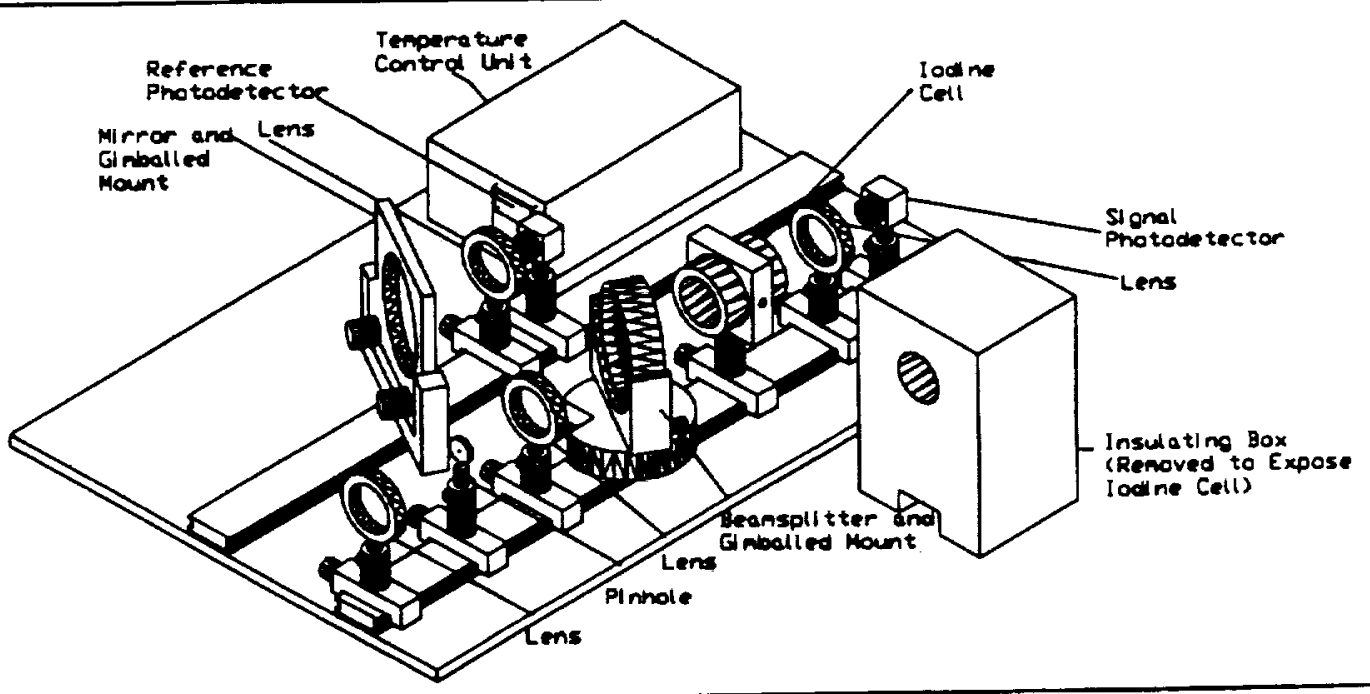

Fig. 3 Apparatus for one PDV chamel 


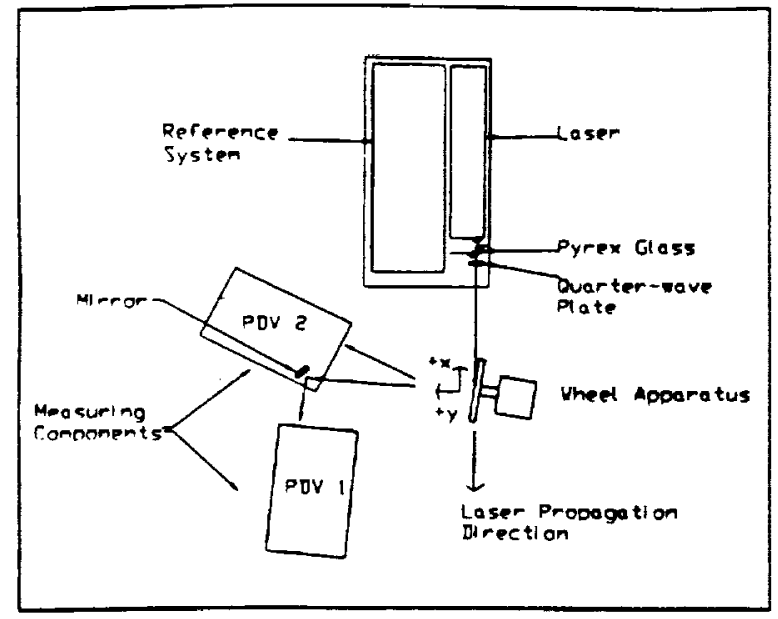

Fig. 4 Schematic of present two-component PDV apparatus, viewed from above, for wheel velocity measurements

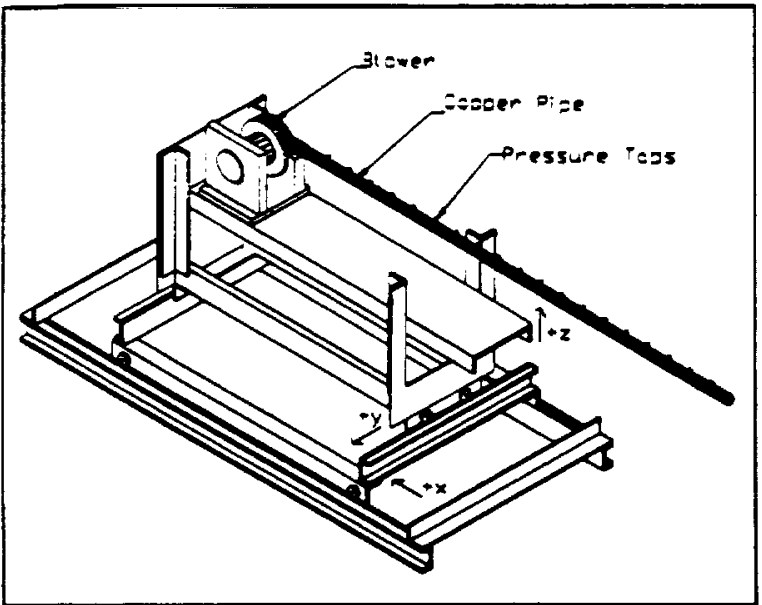

Fig. 5 Throe-axis traverse system, with turbulent pipe flow apparatus installed

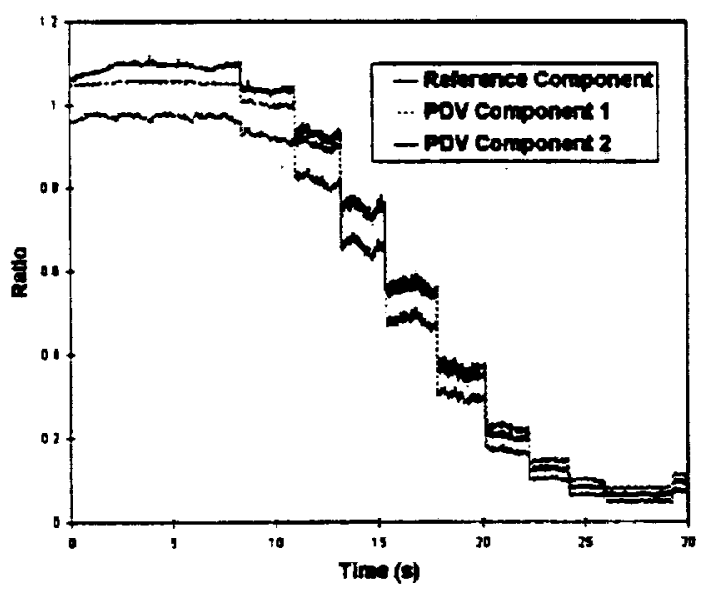

Fig. 6 Example of iodine cell contimuous scan mode hop calibration

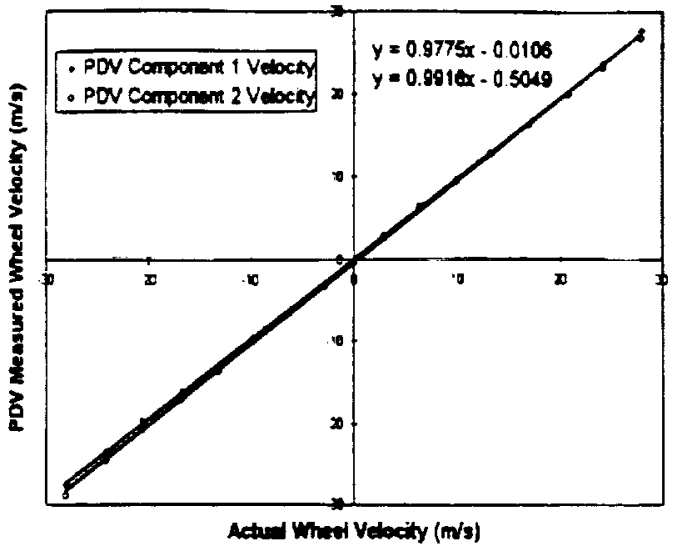

Fig. 7 Typical PDV mean velocity data for rotating wheel: set up with mirror, using known velocity direction

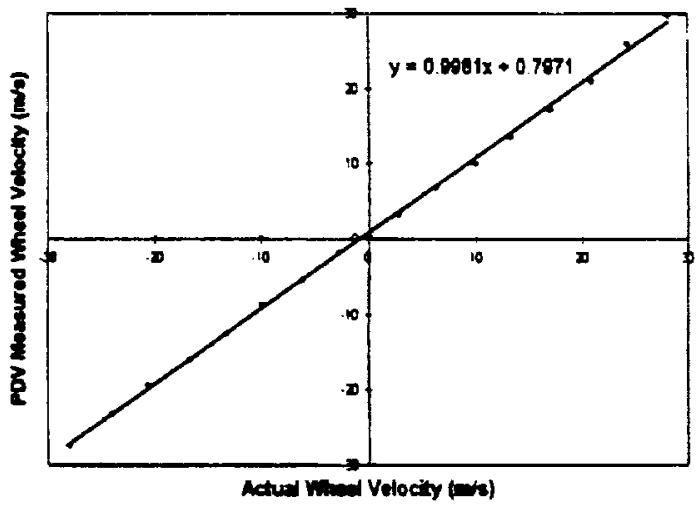

Fig 8 Typical PDV mean velocity data for rotating wheel: two-component set up

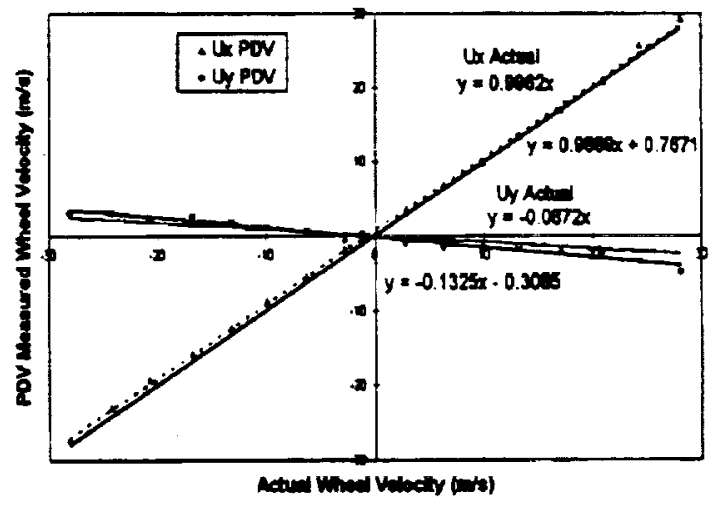

Fig. 9 Resolved $x$ - and y-velocity components used to compute velocity magnitude in Fig. 8 


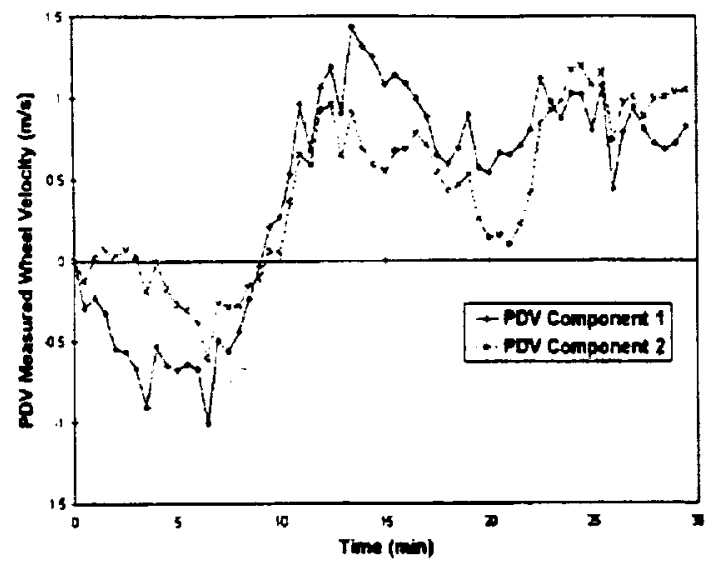

Fig. 10 Measured zero velocity drift over a thirty minute period

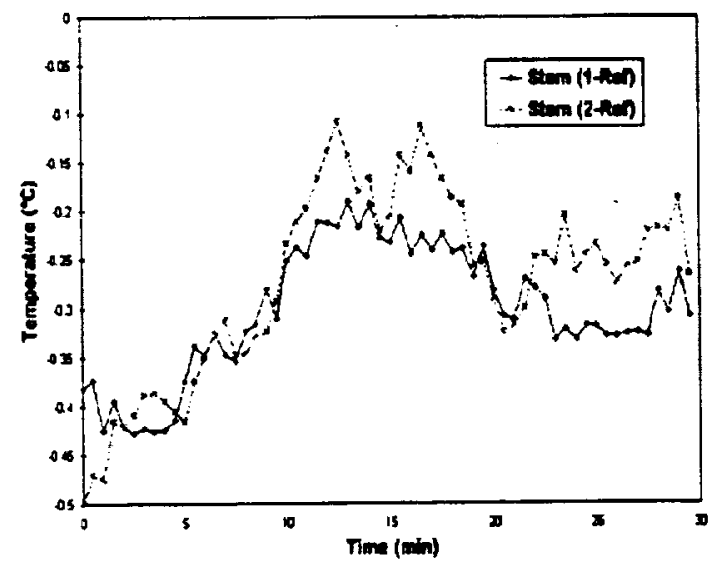

Fig. 11 Differences between signal iodine cell stem temperature and reference cell stem temperature; same time period as in Fig. 10

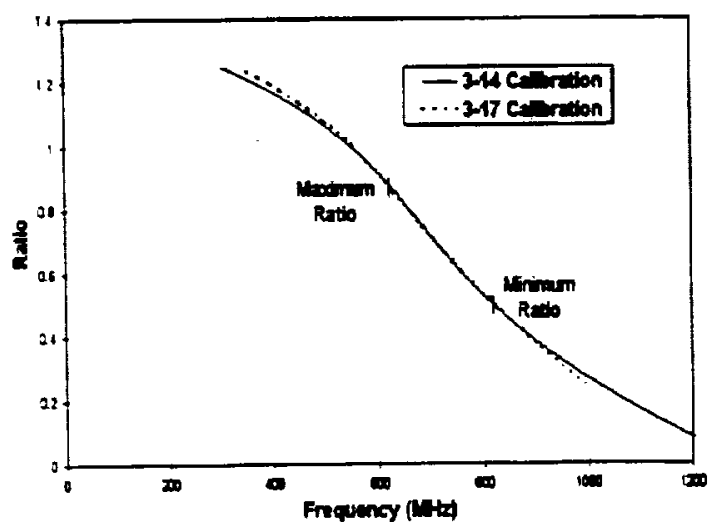

Fig. 12 Example of repeatability of cell calibration curves, used to estimate velocity error due to calibration

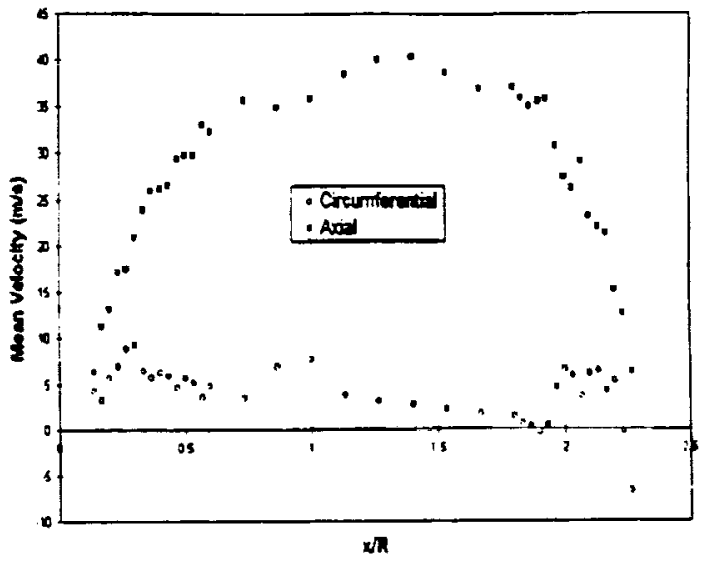

Fig. 13 Preliminary two-component PDV mean velocity profiles in 1.5 inch diameter fully-developed pipe flow

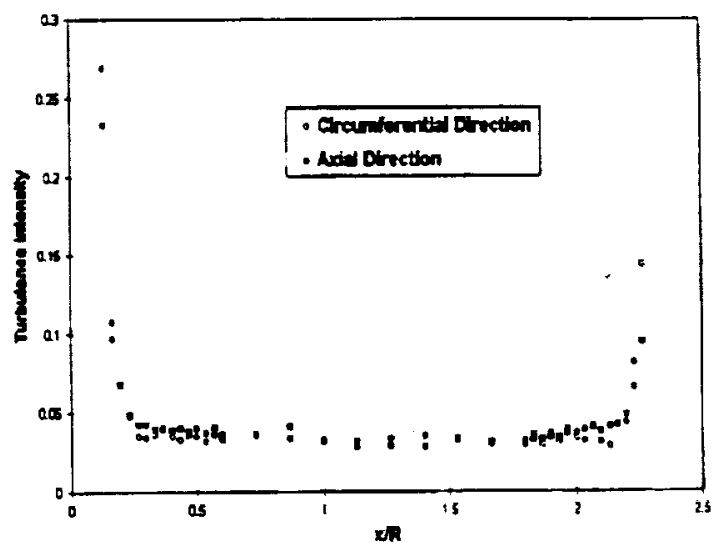

Fig. 14 Preliminary two-component PDV turbulence intensity profiles in 1.5 inch diameter fullydeveloped pipe flow 